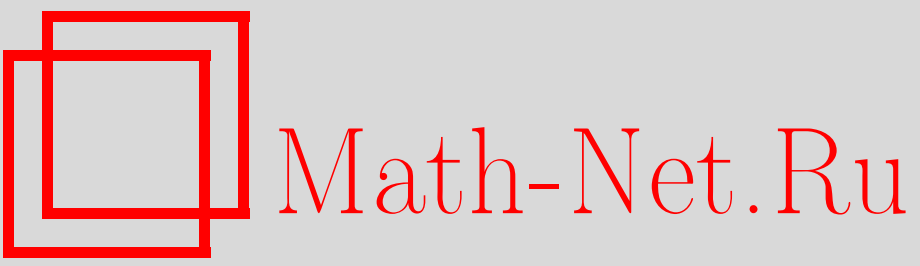

Ю. А. Кашлев, Зависимость равновесных свойств каналированных частиц от поперечной квазитемпературы, ТМФ, 2006, том 146, номер 3, 509-524

DOI: https://doi.org/10.4213/tmf2051

Использование Общероссийского математического портала Math-Net.Ru подразумевает, что вы прочитали и согласны с пользовательским соглашением

http://www.mathnet.ru/rus/agreement

Параметры загрузки:

IP : 54.237 .206 .68

26 апреля 2023 г., 15:43:28 
ТЕОРЕТИЧЕСКАЯ

И МАТЕМАТИЧЕСКАЯ

ФИЗИКА

Том 146, № 3

март, 2006

(C) 2006 г.

Ю. А. Кашлев*

\section{ЗАВИСИМОСТЬ РАВНОВЕСНЫХ СВОЙСТВ КАНАЛИРОВАННЫХ ЧАСТИЦ ОТ ПОПЕРЕЧНОЙ КВАЗИТЕМПЕРАТУРЫ}

Методами неравновесной статистической термодинамики исследованы квазиравновесные и кинетические характеристики каналированных частиц, которые рассматриваются как самостоятельная термодинамическая подсистема. Дан вывод уравнения баланса энергии-импульса каналированных частиц в сопровождающей системе координат. Показано, что решение уравнения баланса дает выражение для основного термодинамического параметра - поперечной квазитемпературы подсистемы каналированных частиц. Исследованы квазиравновесное угловое распределение частиц после прохождения тонкого монокристалла, квазиравновесное распределение по углам выхода частиц в условиях обратного рассеяния, а также константа скорости неравновесного процесса (деканалирования) в условиях большого отклонения системы в целом от термодинамического равновесия. Обсуждается способ измерения поперечной кваизтемпературы пучка по высоте пика углового распределения частиц, полученного в рамках эксперимента "на прострел".

Ключевые слова: неравновесная статистическая термодинамика, каналированные частицы, кристалл, квазиравновесие, поперечная квазитемпература, угловое распределение, минимальный выход.

\section{1. ТЕРМОДИНАМИЧЕСКИЕ ПАРАМЕТРЫ ПОДСИСТЕМЫ КАНАЛИРОВАННЫХ ЧАСТИЦ}

Согласно работе [1] при углах падения быстрых частиц на кристалл, меньших критического угла, пучок частиц, движущихся вдоль кристаллографических плоскостей (осей), распадается на две фракции: каналированную и хаотическую. Образование двух фракций частиц происходит на глубине проникновения $L$ порядка длины когерентности $L_{\mathrm{coh}} \sim 10^{4} \AA[2]$. Поэтому аналитические расчеты, как правило,

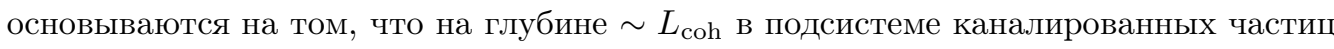
(КЧ) устанавливается квазиравновесное распределение по поперечным энергиям. На этой глубине в уравнении для матрицы плотности КЧ затухают амплитуды вероятности, но сохраняются диагональные матричные элементы, характеризующие

${ }^{*}$ Институт металлургии и материаловедения им. А.А. Байкова РАН, Москва, Россия. E-mail: pm@ultra.imet.ac.ru 


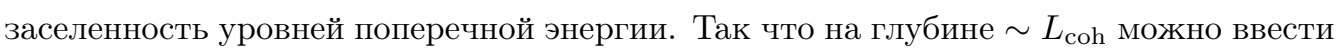
квазиравновесный статистический оператор $\rho_{l}[3],[4]$ и квазиравновесное распределение КЧ.

Если для простоты исключить из рассмотрения хаотическую часть пучка, то остальную часть системы следует разделить еще на две части: $\{i=1\}$ - термостат, включающий кристаллическую решетку и электронный газ, и $\{i=2\}$ - легкие атомные частицы, движущиеся в режиме каналирования. В качестве переменных $P_{m i}$ удобно выбрать гамильтонианы двух подсистем $P_{1 i}=H_{i}$, полные импульсы $P_{2 i}=\mathcal{P}_{i}$ и числа частиц $P_{3 i}=N_{i}$. Поскольку столкновения КЧ с электронами происходят наиболее часто [1], ограничимся учетом электронных столкновений. Тогда гамильтониан КЧ запишется в виде $P_{21}=H_{2}=H_{0}^{(2)}+H_{\text {int }}^{(2)}+\widetilde{H}_{\text {int }}^{(2)}$, где $H_{0}^{(2)}$ включает непрерывный потенциал атомной цепочки $U_{\text {a }}$ (или плоскости), описывающий воздействие на КЧ регулярной решетки в условиях коррелированных столкновений [1]. Полный гамильтониан системы равен $H=H_{1}+H_{2}$. Нами использованы обозначения: $H_{1}=H_{\mathrm{e}}$ - гамильтониан свободных электронов, $H_{\mathrm{int}}^{(2)}=W_{\mathrm{c}}^{(2)}$ - оператор взаимодействия КЧ с одночастичными возбуждениями электронного газа, $\widetilde{H}_{\mathrm{int}}^{(2)}=W_{\mathrm{p}}^{(2)}-$ взаимодействие КЧ с плазмонами. В работе используется система единиц, в которой $\hbar=k_{\mathrm{B}}=1$, а объем системы считается равным единице.

Предположим, что быстрые частицы первоначально двигались со скоростью $V$ вдоль кристаллографической оси в направлении $z$ (или плоскости). Чтобы исключить направленное движение частиц, перейдем в систему координат, сопровождающую частицы.

В соответствии с определениями неравновесной статистической термодинамики [3] оператор энтропии и оператор производства энтропии соответственно равны

$$
\begin{gathered}
S(t, 0)=\Phi+\sum_{m i} P_{m i} F_{i m}(t), \\
\dot{S}(t, 0)=\sum_{m i}\left\{\dot{P}_{m i} F_{i m}(t)+\left(P_{m i}-\left\langle P_{m i}\right\rangle_{l}^{t}\right) \dot{F}_{i m}(t)\right\} .
\end{gathered}
$$

Здесь $\Phi$ - функционал Масье-Планка,

$$
\left\langle P_{m i}\right\rangle_{l}^{t}=\operatorname{Sp}\left(\rho_{l} P_{m i}\right), \quad \rho_{l}=e^{-S(t, 0)} .
$$

Неоператорные величины $F_{i m}(t)$, вошедшие в формулы $(1)$, выбираются таким образом, что функции $F_{i m}(t)$ и средние значения $\left\langle P_{m i}\right\rangle^{t}=\operatorname{Sp}\left(\rho(t) P_{m i}\right)$, вычисленные с использованием неравновесного статистического оператора

$$
\rho(t)=\exp \left\{-S(t, 0)+\int_{-\infty}^{0} d t^{\prime} e^{\epsilon t^{\prime}} \dot{S}\left(t+t^{\prime}, t^{\prime}\right)\right\}, \quad \epsilon \rightarrow+0,
$$

имеют смысл сопряженных термодинамических параметров. Для этого достаточно потребовать выполнения условия соответствия

$$
\left\langle P_{m i}\right\rangle^{t}=\left\langle P_{m i}\right\rangle_{l}^{t} .
$$

Исключим из $\dot{S}(t, 0)(1)$ временнь́е производные $F_{i m}(t)$, используя уравнения

$$
\sum_{n} \frac{\delta^{2} S}{\delta\left\langle P_{m i}\right\rangle_{l}^{t} \delta\left\langle P_{n i}\right\rangle_{l}^{t}}\left\langle\dot{P}_{n i}\right\rangle_{l}^{t}=\dot{F}_{i m}(t)
$$


где $S=\langle S(t, 0)\rangle^{t}$ - энтропия. Тогда, вводя в $\dot{S}(t, 0)(1)$ потоки энергии и импульса КЧ, с учетом выражений (2) и (3) получим

$$
\begin{aligned}
\dot{S}(t, 0)=\Delta & \left.\left(F_{21}-\beta_{1}\right) \dot{H}_{2}-\sum_{\alpha} F_{21} V^{\alpha} \dot{\mathcal{P}}_{2}^{\alpha}-\frac{F_{21}}{m N_{2}} \sum_{\alpha}\left[\mathcal{P}_{2}^{\alpha}-m N_{2} V^{\alpha}\right]\left\langle\dot{P}_{2}^{\alpha}\right\rangle_{l}^{t}\right\}- \\
& -\Delta\left\{\left[\widetilde{H}_{2}-\mu_{2} N_{2}-\frac{N_{2}\left(N_{2}, \widetilde{H}_{2}-\mu_{2} N_{2}\right)^{t}}{\left(N_{2}, N_{2}\right)^{t}}\right]\left\langle\dot{H}_{2}-\sum_{\alpha} V^{\alpha} \dot{\mathcal{P}}_{2}^{\alpha}\right\rangle^{t} \frac{F_{21}^{2}}{C_{2}}\right\} .
\end{aligned}
$$

Здесь использованы следующие обозначения: $\Delta A=A-\langle A\rangle_{l}^{t}, m$ - масса КЧ, $\mu_{2}-$ квазихимический потенциал КЧ,

$$
\widetilde{H}_{2}=H_{2}-\sum_{\alpha} V^{\alpha} \mathcal{P}^{\alpha}+\frac{1}{2} m V^{2} N_{2}, \quad C_{2}=F_{21}^{2}\left(\widetilde{H}_{2}-\mu_{2} N_{2} ; \widetilde{H}_{2}-\mu_{2} N_{2}\right)^{t}
$$

- теплоемкость КЧ, $1 / \beta_{1}$ - температура термостата,

$$
\left.\left(P_{m i}, P_{n j}\right)^{t}=\int_{0}^{1} d \tau \operatorname{Sp}\left\{P_{m i} e^{-\tau S_{0}(t, 0)}\left[P_{n j}-\left\langle P_{n j}\right\rangle\right\rangle_{l}^{t}\right] e^{(\tau-1) S_{0}(t, 0)}\right\}
$$

- корреляционная функция, $i \dot{P}_{m i}=\left[P_{m i}, H\right]$.

Предполагая, что взаимодействие КЧ с электронами является слабым, разложим оператор $\rho(t)$ по степеням потенциала этого взаимодействия, учитывая при этом вид $\dot{S}(t, 0)(4)$. Сохраняя в полученном разложении члены первого порядка, запишем статистический оператор в виде

$$
\begin{aligned}
\rho(t)=\rho_{l}(t) & +\int_{-\infty}^{0} d t^{\prime} e^{\epsilon t^{\prime}} \int_{0}^{1} d \tau e^{-\tau S_{0}(t, 0)} \Delta\left\{\left[F_{21}\left(t+t^{\prime}\right)-\beta_{1}\right] \dot{H}_{2}\left(t^{\prime}\right)-\right. \\
& \left.-\sum_{\alpha} F_{21}\left(t+t^{\prime}\right) V^{\alpha}\left(t+t^{\prime}\right) \dot{\mathcal{P}}_{2}^{\alpha}\left(t^{\prime}\right)\right\} e^{(\tau-1) S_{0}(t, 0)}- \\
& -\int_{0}^{1} d \tau e^{-\tau S_{0}(t, 0)} \Delta\left[\beta_{1}\left(W_{\mathrm{c}}^{(2)}+W_{\mathrm{p}}^{(2)}\right)\right] e^{(\tau-1) S_{0}(t, 0)}
\end{aligned}
$$

где

$$
\begin{gathered}
S_{0}(t, 0)=\Phi_{0}+F_{21}\left(H_{2}-\sum_{\alpha} V^{\alpha} \mathcal{P}_{2}^{\alpha}-\tilde{\mu}_{2} N_{2}\right)+\widetilde{B} \\
\Phi_{0}=\ln \operatorname{Sp} \exp \left\{-F_{21}\left(H_{2}-\sum_{\alpha} V^{\alpha} \mathcal{P}_{2}^{\alpha}-\tilde{\mu}_{2} N_{2}\right)-\widetilde{B}\right\}, \\
\tilde{\mu}_{2}=\mu_{2}-\frac{1}{2} m V^{2}, \quad \widetilde{B}=\beta_{1}\left(H_{1}-\mu_{1} N_{1}\right),
\end{gathered}
$$

$\mu_{1}$ - химический потенциал электронов.

Запишем уравнение баланса для подсистемы $\{2\}$. Изменение направленного импульса КЧ в результате электронных столкновений и, как следствие, рост поперечного импульса происходит за время порядка длительности одного столкновения. За столь короткое время поперечная энергия КЧ не меняется, поскольку ее рост возможен только в результате большого числа столкновений. Рост поперечной энергии 
происходит за время порядка времени релаксации в подсистеме $\{2\}$. Уравнения движения для операторов $H_{2}$ и $\mathcal{P}_{2}$, которые в сопровождающей системе координат включают поперечные составляющие физических величин, могут быть записаны в форме

$$
\begin{gathered}
\dot{H}_{2}=\dot{H}_{2(\mathrm{c})}+n_{2} \sum_{\alpha} \mathcal{F}^{\alpha} V^{\alpha}, \quad \dot{H}_{1}=-\dot{H}_{2}, \\
\dot{\mathcal{P}}_{2}^{\alpha}=\dot{\mathcal{P}}_{2(\mathrm{c})}^{\alpha}+n_{2} \mathcal{F}^{\alpha}, \quad \dot{\mathcal{P}}_{1}=0, \quad \dot{N}_{1}=0,
\end{gathered}
$$

где $n_{2}-$ плотность КЧ, $\alpha=\{x, y\}$,

$$
\dot{H}_{2(\mathrm{c})}=-i\left[H_{0}^{(2)}, W_{\mathrm{c}}^{(2)}\right], \quad \dot{\mathcal{P}}_{2(\mathrm{c})}=-i\left[\mathcal{P}_{2}, W_{\mathrm{c}}^{(2)}\right] .
$$

Индекс "c" в выражениях (7) указывает на то, что изменение величин обусловлено столкновениями КЧ с одночастичными возбуждениями электронного газа. Что же касается взаимодействия с плазмонами, то его можно описать в терминах силы [5]:

$$
\mathcal{F}^{\alpha}=\frac{2 e^{2}}{\pi^{2}} \int d \omega \int d \mathbf{q} \frac{1}{q^{2}} q^{\alpha} \operatorname{Im} \mathcal{E}^{-1}(\mathbf{q}, \omega) \frac{1}{2} \delta\left(\omega-(\mathbf{q} \mathbf{V})-\frac{q^{2}}{2 m}\right),
$$

где $\mathcal{E}(\mathbf{q}, \omega)$ - диэлектрическая функция электронов.

В условиях слабого взаимодействия КЧ с электронами квазитемпература КЧ и температура термостата изменяются настолько медленно, что можно ограничиться стационарным вариантом теории. В этом случае для получения уравнений достаточно усреднить (7) по распределению (6) в условиях стационарности. После усреднения находим уравнение баланса поперечной энергии быстрых частиц и уравнение баланса поперечного импульса частиц:

$$
\begin{array}{r}
\int_{-\infty}^{0} d t^{\prime} e^{\epsilon t^{\prime}}\left(\dot{H}_{2(\mathrm{c})}, \dot{H}_{2(\mathrm{c})}\left(t^{\prime}\right)\right)\left(F_{21}-\beta_{1}\right)+\sum_{\alpha} n_{2} \mathcal{F}^{\alpha} V^{\alpha}- \\
-\sum_{\alpha} F_{21} V^{\alpha} \int_{-\infty}^{0} d t^{\prime} e^{\epsilon t^{\prime}}\left(\dot{H}_{2(\mathrm{c})}, \dot{\mathcal{P}}_{2(\mathrm{c})}^{\alpha}\left(t^{\prime}\right)\right)=0 \\
\int_{-\infty}^{0} d t^{\prime} e^{\epsilon t^{\prime}}\left(\dot{\mathcal{P}}_{2(\mathrm{c})}^{\alpha}, \dot{H}_{2(\mathrm{c})}\left(t^{\prime}\right)\right)\left(F_{21}-\beta_{1}\right)+\mathcal{F}^{\alpha} n_{2}- \\
-\sum_{\beta} F_{21} V^{\beta} \int_{-\infty}^{0} d t^{\prime} e^{\epsilon t^{\prime}}\left(\dot{\mathcal{P}}_{2(\mathrm{c})}^{\alpha}, \dot{\mathcal{P}}_{2(\mathrm{c})}^{\beta}\left(t^{\prime}\right)\right)=0 .
\end{array}
$$

В соответствии с общими принципами кинетической теории [6] отбросим в уравнениях (8) "недиагональные" корреляционные функции, так как они дают вклад более высокого порядка по потенциалу взаимодействия, чем вклад “диагональных" функций. Вместе с тем вклад “диагональных" функций (5) в условиях слабого взаимодействия достаточно вычислить в борновском приближении. С учетом этих приближений на основе уравнений (8), исключая из них силу торможения, находим уравнение баланса энергии-импульса:

$$
n_{2} F_{21} \sum_{\alpha \beta}\left\langle\left(V^{\alpha} V^{\beta}\right)\right\rangle_{0} \mathcal{D}_{p_{\alpha} p_{\beta}}+\left(F_{21}-\beta_{1}\right)\left\langle\left(H_{2}\right)^{2}\right\rangle_{0} \nu_{E_{\perp}}=0 .
$$


Здесь

$$
\begin{aligned}
\langle\ldots\rangle_{0} & =\operatorname{Sp}\left(\rho_{0} \ldots\right), \quad \rho_{0}=e^{-S_{0}(0,0)}, \\
\mathcal{D}_{p_{\alpha} p_{\beta}} & =\frac{1}{n_{2}} \int_{-\infty}^{0} d t^{\prime} e^{\epsilon t^{\prime}}\left(\dot{\mathcal{P}}_{2(\mathrm{c})}^{\alpha}, \dot{\mathcal{P}}_{2(\mathrm{c})}^{\beta}\left(t^{\prime}\right)\right)
\end{aligned}
$$

- диффузионная функция КЧ в пространстве поперечных импульсов,

$$
\nu_{E_{\perp}}=\frac{Q}{\left\langle\left(H_{2}\right)^{2}\right\rangle_{0}}, \quad Q=\int_{-\infty}^{0} d t^{\prime} e^{\epsilon t^{\prime}}\left(\dot{H}_{2(\mathrm{c})}, \dot{H}_{2(\mathrm{c})}\left(t^{\prime}\right)\right)
$$

- частота релаксации поперечной энергии и диссипативная функция КЧ, соответственно. Поскольку уравнение (9) получено в сопровождающей системе координат, вошедший в него параметр $1 / F_{21}$ следует интерпретировать как поперечную квазитемпературу КЧ, т.е. параметр, сопряженный поперечной энергии КЧ.

Стационарная квазитемпература в подсистеме $\{2\}$ устанавливается в результате совместного воздействия, во-первых, диффузии КЧ в пространстве поперечной энергии, во-вторых, диссипативного процесса, обусловленного увлечением электронов частицами, колеблющимися в канале, и, в-третьих, внутренней термализацией подсистемы $\{2\}$, связанной с ангармонизмом колебания частиц [7]. Уравнение баланса (9) включает вклад диффузии (первый член) и диссипации (второй), но полностью не учитывает вклад термализации. Последнее определяется тем, что вклад ангармонизма является настолько малым, что теории каналирования, как правило, строятся в гармоническом приближении. Такая аппроксимация аналогична традиционному приближению теории броуновского движения и теории газов малой плотности, в которых изучаются частицы, взаимодействующие только с атомами среды, но не друг с другом.

Если считать, что частицы падают на решетку под малым углом к цепочке атомов, ориентированной в $z$-направлении, то в режиме каналирования их движение сопровождается осцилляциями в поперечном направлении. Если рассматривать осцилляции в $x$-направлении, то имеется одна диффузионная функция $\mathcal{D}\left(p_{x}\right)$, соответствующая $\alpha=\beta=x$. В этой упрощенной геометрии кинетические функции (10) и (11) были вычислены нами в работе [4]. Подставляя полученные там выражения в уравнение (9), находим окончательный вид уравнения баланса энергии-импульса:

$$
\begin{aligned}
2 \pi n_{2}\left\langle E_{\perp}^{\mathrm{k}}\right\rangle_{0} 4 F_{21} \int_{0}^{\infty} d \omega \int d \mathbf{q} q_{x}^{2}|U(q)|^{2} \operatorname{Im}\left\langle\left\langle\rho_{\mathbf{q}} \mid \rho_{\mathbf{q}}\right\rangle\right\rangle_{\omega} \delta((\mathbf{q} \mathbf{V})-\omega)+ \\
\quad+\frac{1}{4 \pi}\left(F_{21}-\beta_{1}\right)\left\langle E_{\perp}^{2}\right\rangle_{0} \int_{0}^{\infty} d \omega \int d \mathbf{q} \frac{1}{\omega} \operatorname{Im}\left\langle\left\langle f_{\mathbf{q}} \mid f_{\mathbf{q}}^{+}\right\rangle\right\rangle_{\omega} \delta((\mathbf{q} \mathbf{V})-\omega)=0 .
\end{aligned}
$$

Здесь $E_{\perp}^{\mathrm{k}}$ и $E_{\perp}-$ поперечная кинетическая энергия и полная поперечная энергия $\mathrm{K} \Psi, U(q)=e^{2} Z_{1} / q^{2}, \rho_{\mathbf{q}}$ - фурье-компонента флуктуации электронной плотности,

$$
f_{\mathbf{q}}=i \frac{1}{q} \sum_{\mathbf{k q ^ { \prime }}}\left(\mathbf{q q}^{\prime}\right) U\left(q^{\prime}\right) e^{i \mathbf{q}^{\prime} \mathbf{R}}{\stackrel{+}{a_{\mathbf{k}-\mathbf{q}-\mathbf{q}^{\prime}}}}_{a_{\mathbf{k}}}
$$

- фурье-компоненты флуктуационной составляющей силы, действующей на электрон со стороны осциллирующей КЧ [4], $\mathbf{R}$ - радиус-вектор КЧ, ${ }_{\mathbf{k}}^{+}$и $a_{\mathbf{k}}-$ операто- 
ры Ферми, $\left\langle\left\langle\rho_{\mathbf{q}} \mid \rho_{\mathbf{q}}\right\rangle\right\rangle_{\omega}$ и $\left\langle\left\langle f_{\mathbf{q}} \mid f_{\mathbf{q}}\right\rangle\right\rangle_{\omega}-$ фурье-компоненты двухвременнь́х функций Грина плотность-плотность и сила-сила [3].

Теперь выполним в уравнении (12) интегрирование по угловым переменным вектора $q$ и разрешим преобразованное таким образом уравнение относительно $1 / F_{21}$. В результате (после ряда громоздких преобразований) получаем искомое выражение:

$$
\frac{1}{F_{21}}=\frac{1}{\beta_{1}}\left[1+(2 \pi)^{2} K \frac{a}{b}\right] .
$$

Здесь

$$
\begin{aligned}
a= & \int d \sigma(k) k \int d \omega\left[1-\left(\frac{\omega}{k v_{0}}\right)^{2}\right] \frac{4 v_{\mathrm{F}}}{v_{0}} \int_{0}^{\infty} d E\left\{n_{e}(E+\omega)-n_{e}(E)\right\}, \\
b= & \frac{1}{(2 \pi)^{2}} \int d \omega \frac{1}{2 \pi \omega} \frac{1}{v_{\mathrm{F}}} \int_{0}^{\infty} d E\left\{n_{e}(E+\omega)-n_{e}(E)\right\} \times \\
& \times \int d \mathbf{k}^{\prime} \int d \mathbf{k}^{\prime \prime}\left|U\left(k^{\prime \prime}\right)\right|^{2} \frac{\left(\mathbf{k}^{\prime} \mathbf{k}^{\prime \prime}\right)^{2}}{\left(k^{\prime}\right)^{2}\left|\mathbf{k}^{\prime}+\mathbf{k}^{\prime \prime}\right|} \delta\left(\left(\mathbf{k}^{\prime} \mathbf{v}_{0}\right)+\frac{\left(k^{\prime}\right)^{2}}{2 m}-\omega\right) .
\end{aligned}
$$

Кроме того, при записи выражений (13), (14) использованы следующие обозначения:

$$
\begin{gathered}
K=\frac{\left\langle E_{\perp}\right\rangle_{0}}{\left\langle E_{\perp}^{2}\right\rangle_{0}} \\
d \sigma(k)=\frac{1}{2 \pi}|U(k)|^{2} \frac{1}{v_{\mathrm{F}}^{2}} k d k
\end{gathered}
$$

- сечение упругого рассеяния, $v_{\mathrm{F}}$ - скорость Ферми, $n_{e}(E)$ - функция распределения Ферми, $v_{0}$ - скорость КЧ в $z$-направлении.

Будем рассматривать электроны как вырожденный газ при нулевой температуре. В этой модели при $v_{0}>v_{\mathrm{F}}$ после интегрирования в выражениях (14) по $d \omega, d E, d \mathbf{k}^{\prime}$ и подстановки полученных выражений в (13) имеем

$$
\frac{1}{F_{21}}=\frac{1}{\beta_{1}}\left(1+20 \pi^{2} K \frac{\varepsilon_{\mathrm{F}}}{k_{\mathrm{F}}^{3}} \frac{I_{1}}{I_{2}}\right),
$$

где

$$
\begin{gathered}
I_{1}=\int d \sigma(\theta)\left(\sin \frac{\theta}{2}\right)^{3}, \quad I_{2}=\int d \sigma(\theta)\left(\sin \frac{\theta}{2}\right)^{5 / 2} I_{3}(\theta), \quad \varepsilon_{\mathrm{F}}=\frac{1}{2} m v_{\mathrm{F}}^{2}, \\
I_{3}(\theta)=\int d \cos \varphi\left(\cos ^{2} \varphi+\frac{4}{3}\right)\left(\cos \varphi+\sin \frac{\theta}{2}\right)^{-1 / 2}, \quad \cos \theta=\frac{\left(\mathbf{k}^{\prime} \mathbf{k}^{\prime \prime}\right)}{k^{\prime} k^{\prime \prime}}, \quad k_{\mathrm{F}}=m v_{\mathrm{F}} .
\end{gathered}
$$

\section{2. УГЛОВОЕ РАСПРЕДЕЛЕНИЕ КЧ ПОСЛЕ ПРОХОЖДДЕНИЯ ЧЕРЕЗ ТОНКИЙ КРИСТАЛЛ И ЕГО ЗАВИСИМОСТЬ ОТ ПОПЕРЕЧНОЙ КВАЗИТЕМПЕРАТУРЫ КЧ}

При движении быстрых частиц в осевом канале их скорость направлена под малым углом к кристаллографической оси. Поперечную кинетическую энергию частиц на входе и выходе из тонкого кристалла обозначим $\tau_{\perp}^{\text {in }}$ и $\tau_{\perp}^{\text {out }}$. Пусть площадь 
базисной ячейки канала, которая лежит внутри контура поперечной потенциальной энергии $\Phi_{\perp}$, равна $f\left(\Phi_{\perp}\right)$, причем энергия $\Phi_{\perp}$ не превышает критического значения $\Phi_{h}$, а контур критической потенциальной энергии $\Phi_{h}$ проходит через седловую точку потенциального рельефа канала. Долю площади ячейки в поперечной плоскости внутри контура $\Phi_{h}$ обозначим $f_{h}$.

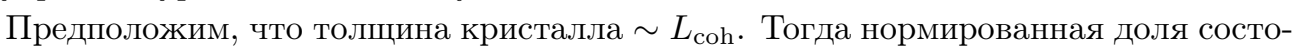
яний КЧ с полной поперечной энергией на выходе $E_{\perp}^{\text {out }}$ и поперечной кинетической энергией в интервале $\left(\tau_{\perp}^{\text {out }}, \tau_{\perp}^{\text {out }}+d \tau_{\perp}^{\text {out }}\right)$ равна

$$
\frac{1}{f\left(E_{\perp}^{\text {out }}\right)} f^{\prime}\left(E_{\perp}^{\text {out }}-\tau_{\perp}^{\text {out }}\right) d \tau_{\perp}^{\text {out }}
$$

Здесь использовано обозначение

$$
f^{\prime}\left(E_{\perp}^{\text {out }}-\tau_{\perp}^{\text {out }}\right)=\left.\frac{d}{d \Phi_{\perp}} f\left(\Phi_{\perp}\right)\right|_{\Phi_{\perp}=E_{\perp}^{\text {out }}-\tau_{\perp}^{\text {out }}}
$$

Будем считать, что при прохождении через кристалл поперечная энергия КЧ возрастает на $\varepsilon_{\perp}$ за счет электронного рассеяния. Тогда доля состояний частиц с начальной энергией $\tau_{\perp}^{\text {in }}$, которые получили дополнительную энергию $\varepsilon_{\perp}$ и вышли из кристалла, имея энергии в интервалах $\left(\tau_{\perp}^{\text {out }}, \tau_{\perp}^{\text {out }}+d \tau_{\perp}^{\text {out }}\right)$ и $\left(E_{\perp}^{\text {out }}, E_{\perp}^{\text {out }}+d E_{\perp}^{\text {out }}\right)$, равна

$$
G\left(E_{\perp}^{\text {out }}, \tau_{\perp}^{\text {out }}, \tau_{\perp}^{\text {in }}, \varepsilon_{\perp}\right) d \tau_{\perp}^{\text {out }} d E_{\perp}^{\text {out }}=\frac{f^{\prime}\left(E_{\perp}^{\text {out }}-\tau_{\perp}^{\text {out }}\right)}{f\left(E_{\perp}^{\text {out }}\right)} f^{\prime}\left(E_{\perp}^{\text {out }}-\tau_{\perp}^{\text {in }}-\varepsilon_{\perp}\right) d \tau_{\perp}^{\text {out }} d E_{\perp}^{\text {out }} .
$$

Когда подсистема КЧ достигает квазиравновесия на глубине $\sim L_{\mathrm{coh}}$, вероятность того, что дополнительная энергия $\varepsilon_{\perp}$ лежит в интервале $\left(\varepsilon_{\perp}, \varepsilon_{\perp}+d \varepsilon_{\perp}\right)$, имеет вид [8]

$$
p\left(\varepsilon_{\perp}\right)=g\left(\varepsilon_{\perp}\right) n\left(\varepsilon_{\perp}\right)
$$

где

$$
\begin{aligned}
& n\left(\varepsilon_{\perp}\right)=\lambda e^{-F_{21} \varepsilon_{\perp},} \\
& g\left(\varepsilon_{\perp}\right)=f^{\prime}\left(\varepsilon_{\perp}-\tau_{\perp}\right)
\end{aligned}
$$

- плотность состояний КЧ, $\lambda=e^{F_{21} \mu_{2}}$ - абсолютная активность КЧ. Абсолютная активность при высоких квазитемпературах КЧ (см. таблицу в приложении) является слабо меняющейся функцией и, если распределение (16) нормировано на единицу, ее можно заменить предельным значением $\lambda=1$ при $1 / F_{21} \rightarrow \infty$. Усредняя нормированную долю состояний по распределению (16), получаем

$$
\begin{gathered}
\left\langle G\left(E_{\perp}^{\text {out }}, \tau_{\perp}^{\text {out }}, \tau_{\perp}^{\text {in }} ; \frac{1}{F_{21}}\right)\right\rangle d \tau_{\perp}^{\text {out }} d E_{\perp}^{\text {out }}=\frac{f^{\prime}\left(E_{\perp}^{\text {out }}-\tau_{\perp}^{\text {out }}\right)}{f\left(E_{\perp}^{\text {out }}\right)} \times \\
\times \int_{0}^{\infty} d \varepsilon_{\perp} p\left(\varepsilon_{\perp}\right) f^{\prime}\left(E_{\perp}^{\text {out }}-\tau_{\perp}^{\text {in }}-\varepsilon_{\perp}\right) d \tau_{\perp}^{\text {out }} d E_{\perp}^{\text {out }}
\end{gathered}
$$

Теперь введем интегральное распределение КЧ. Будем считать, что эксперимент "на прострел" выполняется в условиях $\tau_{\perp}^{\text {out }}=\tau_{\perp}^{\text {in }}$. Кроме того, будем использовать линейное приближение, записывая часть площади базисной ячейки в виде $f\left(\Phi_{\perp}\right)=$ 
$\left(f_{h} / \Phi_{h}\right) \Phi_{\perp}[9],[10]$. В этом приближении с учетом $(16)$ интегральное распределение частиц равно

$$
\begin{aligned}
\frac{1}{\Phi_{h}} \mathcal{F}\left(\Phi_{h}, \tau_{\perp}^{\text {out }} ; \frac{1}{F_{21}}\right) & \equiv \int_{0}^{\Phi_{h}} d E_{\perp}^{\prime}\left\langle G\left(E_{\perp}^{\prime}, \tau_{\perp}^{\text {out }}, \tau_{\perp}^{\text {out }} ; \frac{1}{F_{21}}\right)\right\rangle= \\
& =\frac{f_{h}}{\Phi_{h}}\left[\ln \left(\frac{\Phi_{h}}{\tau_{\perp}^{\text {out }}}\right)-e^{F_{21} \tau_{\perp}^{\text {out }}} \Gamma\left(0, F_{21} \tau_{\perp}^{\text {out }}\right)\right]
\end{aligned}
$$

где $\Gamma(a, x)$ - неполная гамма-функция. Поскольку $\tau_{\perp}^{\text {out }}=E_{0} \theta_{\text {out }}^{2}$, где $E_{0}$ - энергия падающей частицы, формула (18) определяет распределение частиц по углам выхода $\theta_{\text {out }}$ в геометрии $\theta_{\text {in }}=\theta_{\text {out }}$.

Используя формулу (18), вычислим высоту пика углового распределения частиц, выходящих с обратной стороны кристалла толщиной $\sim L_{\text {соһ }}$. Для расчета высоты необходимо принять во внимание тот факт, что кристаллическая пластина состоит из отдельных блоков и имеет мозаичную структуру с конечным углом разупорядочения блоков $\psi_{m}$. Учитывая гауссовское распределение блоков, после усреднения выражения (18) по мозаичной структуре запишем выражение для высоты пика в виде

$$
I\left(\theta=0 ; \frac{1}{F_{21}}\right)=\frac{\psi_{\mathrm{det}}^{2}}{\psi_{h}^{2}} \int_{0}^{\infty} d \varphi \varphi \mathcal{F}\left(\Phi_{h}, E_{0} \varphi^{2} ; \frac{1}{F_{21}}\right) \frac{1}{2 \psi_{m}^{2}} e^{-\varphi^{2} /\left(2 \psi_{m}^{2}\right)} .
$$

Здесь $\Phi_{h}=E_{0} \psi_{h}^{2}, \quad \psi_{\text {det }}^{2} / \psi_{h}^{2}$ - геометрический фактор, $\pi \psi_{h}^{2}$ - предельное значение телесного угла. Подставим выражение $(18)$, где $\tau_{\perp}^{\text {out }}=E_{0} \varphi^{2}$, в высоту пика (19) и проинтегрируем по $d \varphi$. В результате высоту углового распределения удается представить как функцию поперечной квазитемпературы КЧ:

$$
I\left(\theta=0 ; \frac{1}{F_{21}}\right)=f_{h} \frac{\psi_{\mathrm{det}}^{2}}{\psi_{h}^{2}}\left[0.28+0.5 \ln \left(\frac{\Phi_{h}}{2 E_{0} \psi_{m}^{2}}\right)+\left(2-4 F_{21} E_{0} \psi_{m}^{2}\right)^{-1} \ln \left(2 F_{21} E_{0} \psi_{m}^{2}\right)\right] .
$$

Угловое распределение частиц имеет гауссовскую форму [11], [12]. Поэтому можно записать

$$
I\left(\theta ; \frac{1}{F_{21}}\right)=I\left(0 ; \frac{1}{F_{21}}\right) \exp \left\{-\frac{1}{1 / F_{21}} E_{0}\left[\theta-\sqrt{\left(\Delta \theta^{2}\right)_{e}}\right]^{2}\right\} .
$$

Здесь

$$
\left(\Delta \theta^{2}\right)_{e}=\frac{m_{e}}{2 m E_{0}}\left(-\frac{d E}{d x}\right)_{e} L_{\mathrm{coh}}
$$

- приращение квадрата флуктуации угла за счет электронных столкновений на глубине когерентности, $m_{e}$ - масса электрона, $(-d E / d x)_{e}-$ электронные энергетические потери частиц.

На рис. 1 представлено угловое распределение ионов йода после прохождения кристалла серебра (все параметры даны в приложении), вычисленное на основе формул (20)-(22). Как видно из рис. 1, форма теоретической кривой удовлетворительно согласуется с данными эксперимента (точки).

Сделаем еще два замечания. Во-первых, зависимость распределения (21) от квазитемпературы (15) определяется двумя факторами: экспоненциальным множителем, который растет с ростом $1 / F_{21}$, и высотой пика $(20)$, которая уменьшается 


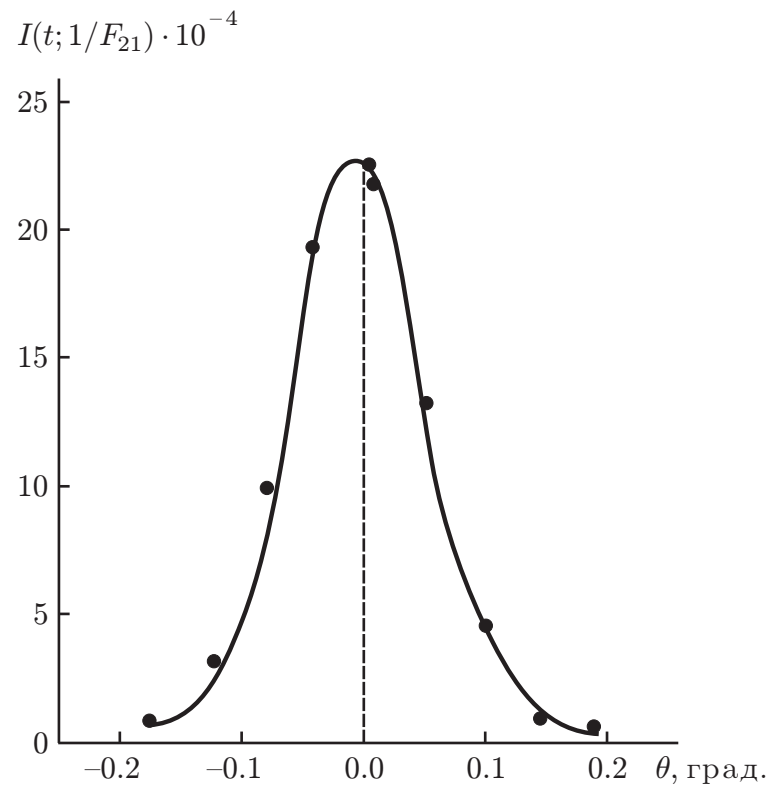

Рис. 1.

при увеличении $1 / F_{21}$. Во-вторых, гауссовское распределение соответствует случаю $\theta_{\text {in }}=\theta_{\text {out }}$. Однако возможна и более сложная геометрия эксперимента "на прострел", a именно, когда $\theta_{\text {in }} \neq \theta_{\text {out }}$ и направление выхода пучка совпадает с направлением атомной цепочки одной из кристаллографических плоскостей кристалла [13]. Тогда угловое распределение имеет два пика [13]: большой максимум в направлении $\theta_{\text {in }}=\theta_{\text {out }}$ и малый - в направлении цепочки. Последний связан с эффектом вовлечения первоначально неканалированных частиц в режим направленного движения под воздействием потенциала плоскости. Угловое распределение частиц, вовлеченных в режим, гауссовское.

Используя линейное приближение для $f\left(\Phi_{\perp}\right)[9],[10]$, вычислим величину $K$, вошедшую в выражение (15). Для осевого канала в пределе $1 / F_{21} \rightarrow \infty$ имеем

$$
K=\frac{\int_{0}^{\Phi_{h}} d \varepsilon_{\perp} \varepsilon_{\perp} \frac{d f\left(\varepsilon_{\perp}-\tau_{\perp}\right)}{d \varepsilon_{\perp}}}{\int_{0}^{\Phi_{h}} d \varepsilon_{\perp} \varepsilon_{\perp}^{2} \frac{d f\left(\varepsilon_{\perp}-\tau_{\perp}\right)}{d \varepsilon_{\perp}}}=\frac{3}{2} \frac{1}{\Phi_{h}} .
$$

\section{3. РАСПРЕДЕЛЕНИЕ ЧАСТИЦ ПО УГЛАМ ВЫХОДА В УСЛОВИЯХ ОБРАТНОГО РАССЕЯНИЯ. ЗАВИСИМОСТЬ РАСПРЕДЕЛЕНИЯ ОТ ПОПЕРЕЧНОЙ КВАЗИТЕМПЕРАТУРЫ КЧ}

Будем считать, что атомные цепочки, образующие осевой канал, "заморожены” и среднеквадратичный разброс атомов в поперечной плоскости равен $\sigma$. Введем следующие обозначения: $\theta$ - угол между направлением скорости КЧ и осью цепочки, $\rho^{\prime}-$ расстояние частицы от оси цепочки в поперечной плоскости. Статистическая 
механистическая теория [14] установила связь углового распределения $\tilde{I}(\theta)$ по поперечным кинетическим энергиям $E_{\perp}^{\mathrm{k}}=E_{0} \theta^{2}$ с распределением по полным поперечным энергиям $\widetilde{P}\left(E_{\perp}\left(\theta, \rho^{\prime}\right)\right)$ :

$$
\tilde{I}(\theta)=2 \int_{0}^{\rho_{0}} d \rho^{\prime} \rho^{\prime} \frac{\widetilde{P}\left(E_{\perp}\left(\theta, \rho^{\prime}\right)\right)}{\rho_{0}^{2}-\Lambda^{2}\left(\rho^{\prime}\right)} .
$$

Здесь $E_{\perp}\left(\theta, \rho^{\prime}\right)=E_{\perp}^{\mathrm{k}}+U_{\mathrm{a}}\left(\rho^{\prime}\right), \quad \rho_{0}=(\pi N d)^{-1 / 2}-$ радиус области, приходящейся на одну атомную цепочку в поперечной плоскости, $N$ - плотность атомов решетки, $d-$ расстояние между атомами цепочки. Величина $\Lambda\left(\rho^{\prime}\right)$, как показано в работе [14], определяется из условия $U_{\mathrm{a}}(\Lambda)=E_{\perp}$.

Вычислим плотность состояний частиц в задаче обратного рассеяния. Разделяя составляющие радиус-вектора и скорости частиц $(\rho, z)$ и $\left(v_{\perp}, v_{z}\right)$, запишем плотность вероятности нахождения частиц в интервале $(\rho, \rho+d \rho)$ со скоростями в интервалах $\left(v_{z}, v_{z}+d v_{z}\right)$ и $\left(v_{\perp}, v_{\perp}+d v_{\perp}\right)$. Пусть $\tilde{d}(\theta)$ - глубина, начиная с которой на частицу действует усредненный потенциал цепочки $U_{\mathrm{a}}$ [1]. Тогда, учитывая, что на малой глубине (в приповерхностном слое) плотность состояний имеет гауссовскую форму [15], ее нетрудно записать на большей глубине, выполнив в выражении [15] замену $\rho^{\prime}$ на $\rho^{\prime}+\left(v_{\perp} \tilde{d}(\theta) / v_{z}\right)$. Принимая во внимание, что в задачах обратного рассеяния параметр $\tilde{d}(\theta)$ можно заменить его асимптотическим значением $d / 2$ [1], получаем

$$
\begin{aligned}
g\left(\rho^{\prime}, v_{\perp}, v_{z}, \theta \mid z=\frac{d}{2}\right)=( & \left.2 \pi \sigma^{2} \cdot 2 \pi v_{0} \sin \theta\right)^{-1} \times \\
& \times \exp \left\{-\frac{1}{2 \sigma^{2}}\left(\rho^{\prime}-\frac{v_{\perp} d}{2 v_{z}}\right)^{2}\right\} \delta\left(v_{\perp}-v_{0} \sin \theta\right) \delta\left(v_{z}-v_{0} \cos \theta\right) .
\end{aligned}
$$

Перейдем к плотности состояний в переменных $\theta, \rho^{\prime}$. Для этого $g\left(\rho^{\prime}, v_{\perp}, v_{z}, \theta \mid d / 2\right)$ следует проинтегрировать по $d v_{\perp}, d v_{z}$. Запишем полученное таким образом выражение в безразмерной форме:

$$
g\left(E_{\perp}\left(\theta, \rho^{\prime}\right)\right)=\sigma^{-2} \int_{\Lambda\left(\rho^{\prime}\right)}^{\rho_{0}} d \rho \rho \exp \left\{-\frac{1}{2}\left[B^{2}\left(\theta, \rho, \rho^{\prime}\right)+\left(\frac{\rho}{\sigma}\right)^{2}\right]\right\} J_{0}\left(i z^{\prime}\right),
$$

где

$$
B^{2}\left(\theta, \rho, \rho^{\prime}\right)=\left(\frac{d}{2 \sigma}\right)^{2} \frac{1}{E_{0}}\left\{E_{\perp}\left(\theta, \rho^{\prime}\right)-U_{\mathrm{a}}(\rho)\right\}, \quad z^{\prime}=\frac{\rho}{\sigma} B\left(\theta, \rho, \rho^{\prime}\right),
$$

$J_{0}(x)$ - функция Бесселя.

Имея явный вид плотности (25), нетрудно обобщить угловое распределение на базе определений статистической термодинамики. С этой целью введем среднее число заполнения состояния с энергией

$$
n\left(E_{\perp}\left(\theta, \rho^{\prime}\right)\right)=\lambda e^{-F_{21} E_{\perp}\left(\theta, \rho^{\prime}\right)}
$$

(формула аналогична (16)). Используя (25) и (26), представим искомое угловое распределение как функцию поперечной квазитемпературы:

$$
\tilde{I}\left(\theta ; \frac{1}{F_{21}}\right)=2 \int_{0}^{\rho_{0}} d \rho^{\prime} \rho^{\prime} \frac{1}{\rho_{0}^{2}-\Lambda^{2}\left(\rho^{\prime}\right)} g\left(E_{\perp}\left(\theta, \rho^{\prime}\right)\right) n\left(E_{\perp}\left(\theta, \rho^{\prime}\right)\right) .
$$


Преобразуем выражение (27). Перейдем к безразмерным переменным

$$
\begin{array}{lll}
\zeta=\frac{\rho}{a_{0}}, & \zeta_{0}=\frac{\rho_{0}}{a_{0}}, \quad \xi=\frac{\rho^{\prime}}{a_{0}}, & \eta=\frac{a_{0}^{2}}{2 \sigma^{2}}, \\
\psi=\frac{\theta}{\psi_{1}}, & \varkappa^{2}=\left(\frac{d^{2}}{8 \sigma^{2}}\right)\left(\frac{U_{0}}{E_{0}}\right), & \gamma=\frac{R_{\mathrm{eff}}}{a_{0}},
\end{array}
$$

где $a_{0}$ - радиус экранирования, $U_{0}$ - высота потенциального барьера в случае осевого канала, $\psi_{1}$ - критический угол каналирования [1], $R_{\mathrm{eff}}=\sqrt{a_{0}^{2}+2 \sigma^{2}}-$ эффективный радиус действия с учетом смещения атома решетки из узла на расстояние $\sigma$. Далее запишем потенциал цепочки в виде $U_{\mathrm{a}}(\zeta)=U_{0} w(\zeta)$ и в первом приближении аппроксимируем $w(\zeta)$ ступенчатой функцией $\theta(\gamma-\zeta)$. После перехода к безразмерным переменным подставим (25) в (27). В результате преобразований распределение обратно рассеянных частиц, которые первоначально (в режиме каналирования) достигли глубины $\sim L_{\mathrm{coh}}$, принимает вид

$$
\tilde{I}\left(\psi ; \frac{1}{F_{21}}\right)=2 \lambda \eta \frac{1}{\zeta_{0}^{2}} e^{-F_{21} U_{0} \psi^{2}}\left\{\gamma^{2} e^{-F_{21} U_{0}} R_{1}(\psi)+2 \widetilde{R}_{0}(\psi)\right\},
$$

где

$$
\begin{aligned}
R_{n}(\psi)= & \int_{0}^{\zeta_{0}} d \zeta \zeta \Phi_{n}(\psi, \zeta), \\
\widetilde{R}_{n}(\psi)= & \int_{\gamma}^{\zeta_{0}} d \xi \xi\left[\zeta_{0}-\zeta_{1}(\psi, \xi)\right]^{-1} \int_{\zeta_{1}(\psi, \xi)}^{\zeta_{0}} d \zeta \zeta \Phi_{n}(\psi, \zeta), \\
\Phi_{n}(\psi, \zeta)= & \exp \left\{-\left[\eta \zeta^{2}+\varkappa^{2}\left(\psi^{2}-w(\zeta)+n\right)\right]\right\} \sum_{m=0}^{\infty} \varkappa^{2 m}(m !)^{-2} \times \\
& \times\left[\eta \zeta^{2}\left(\psi^{2}-w(\zeta)+n\right)\right]^{m} .
\end{aligned}
$$

Функция $\Phi_{n}(\psi, \zeta)$ в выражении $(28)$ представлена в виде ряда по степеням $\varkappa^{2}$; поскольку в области высоких энергий $\varkappa^{2} \ll 1$, индекс принимает два значения: $n=0,1$. Величина $\zeta_{1}(\psi, \xi)$ в $(28)$ определена формулой

$$
w\left(\zeta_{1}(\psi, \xi)\right)= \begin{cases}\psi^{2}+w(\xi), & \psi^{2}+w(\xi) \leqslant 1 \\ 0, & \psi^{2}+w(\xi)>1\end{cases}
$$

Сохраним в разложении функции $\Phi_{n}(\psi, \zeta)$ только первый член. Затем проинтегрируем $R_{n}(\psi)$ и $\widetilde{R}_{n}(\psi)$ по $d \xi, d \zeta$ и подставим полученные выражения в распределение (28). В результате находим, что минимальный выход обратно рассеянных частиц $\chi_{\min }\left(1 / F_{21}\right)=\tilde{I}\left(\psi=0 ; 1 / F_{21}\right)$ в реальных физических условиях $\eta \zeta_{0}^{2} \gg 1$ зависит от поперечной квазитемпературы (15) следующим образом:

$$
\chi_{\min }\left(\frac{1}{F_{21}}\right)=\lambda\left(\frac{\gamma}{\zeta_{0}}\right)^{2} e^{-F_{21} U_{0}}
$$

Как показано в работе [8], для перехода от выражений статистической термодинамики к формулам теории Линдхарда [1] достаточно выполнить предельный переход 


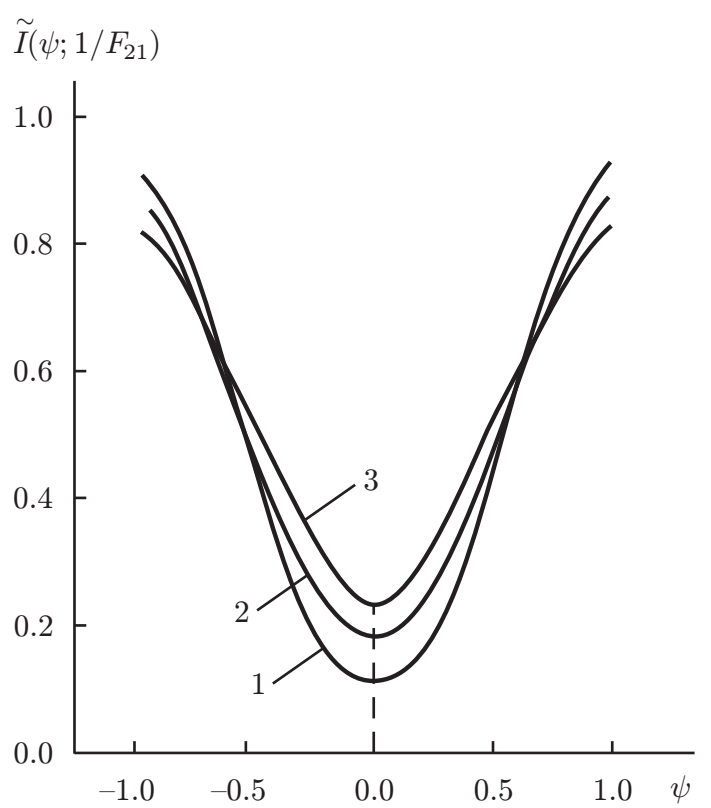

Рис. 2.

$1 / F_{21} \rightarrow \infty$. В этом пределе формула $(29)$ дает

$$
\chi_{\min }=\left(\frac{\gamma}{\zeta_{0}}\right)^{2}=\frac{a_{0}^{2}+2 \sigma^{2}}{\rho_{0}^{2}},
$$

что полностью совпдает с выражением, полученным в работе [1].

В широком интервале углов расчет распределения обратно рассеянных частиц возможен на базе формулы (28) и методов численного интегрирования. Результат такого расчета показан на рис. 2, где представлено угловое распределение протонов с энергией 1.6 МэВ, первоначально прошедших в осевом канале [110] кристалла кремния. Кривые на рис. 2 соответствуют трем значениям квазитемпературы (15), а именно: 1) $\left.\left.1 / F_{21}=U_{0} / 3,2\right) 1 / F_{21}=2 U_{0} / 3,3\right) 1 / F_{21}=U_{0}$. Согласно рис. 2 минимум углового распределения убывает с уменьшением $1 / F_{21}$, что полностью согласуется с результатом аналитической теории (29).

\section{4. КОНСТАНТА СКОРОСТИ НЕОБРАТИМОГО ПРОЦЕССА В СИЛЬНО НЕРАВНОВЕСНОЙ СИСТЕМЕ, ЕЕ ЗАВИСИМОСТЬ ОТ КВАЗИТЕМПЕРАТУРЫ}

В работе [8] было установлено, что на глубине проникновения $\sim L_{\mathrm{coh}}$, несмотря на достижение внутреннего равновесия в подсистеме КЧ, система в целом остается сильно неравновесной с большим отрывом термодинамических параметров отдельных подсистем.

Первичными процессами прохождения КЧ через кристалл являются акты рассеяния частиц на электронах и фононах. Каждый акт сопровождается переходом 
КЧ между уровнями поперечной энергии. Если в процессе случайных блужданий по уровням КЧ достигает уровня $\omega_{s_{0}}$, соответствующего потенциалу захвата частицы в режим каналирования, то она переходит в хаотическую часть пучка, т.е. деканалирует. Акт деканалирования можно описать с помощью “квазихимической” реакции вида

$$
\left\{\begin{array}{l}
S\left\{A_{1}\right\}+A_{2} \underset{k_{-1}}{\stackrel{k_{1}}{\rightleftarrows}} S\left\{A_{1}\right\}+\stackrel{*}{A_{2}}, \\
\stackrel{*}{A_{2}} \stackrel{k_{2}}{\longrightarrow} A_{3},
\end{array}\right.
$$

где символ $A_{i}$ соответствует частицам $i$-й подсистемы, а $S\left\{A_{1}\right\}$ - совокупности атомов термостата. Согласно реакции (30), деканалирование помимо перехода КЧ на уровень $\omega_{s_{0}}\left(\right.$ константа скорости $\left.k_{1}\right)$ включает обратный процесс - релаксацию с константой скорости $k_{-1}$ и квазихимическое превращение $\{2\} \rightarrow\{3\}$ с константой $k_{2}$. Поскольку плотность атомов термостата на много порядков выше плотности КЧ, можно утверждать [16], что стационарное течение реакции (30) наступает достаточно быстро. Причем в стационарном режиме переходы $\{2\} \rightarrow\{3\}$ и релаксация уравновешиваются образованием возбужденных частиц по реакции с константой скорости $k_{1}$. Поскольку термодинамические параметры термостата значительно отличаются от параметров КЧ (оценка квазитемпературы (15), (23) в случае каналирования ионов йода в кристалле серебра дает $1 / F_{21} \sim 5$ эВ), в условиях уравновешивания, указанного выше, выполняется соотношение $k_{1} / k_{-1}=e^{-F_{21} \omega_{s_{0}}}$.

Для КЧ релаксация с константой скорости $k_{-1}$ предпочтительнее резонансного перехода $\{2\} \rightarrow\{3\}$ (резонанс по энергиям), поэтому $k_{2} \ll k_{-1}$. Учитывая это условие и соотношение химической кинетики для двухстадийной реакции [16], можно записать константу скорости реакции (30) в следующем виде:

$$
k_{\mathrm{tot}}=k_{2}\left(\frac{k_{-1}}{k_{1}}+1\right)^{-1} .
$$

Подставляя явный вид отношения $k_{1} / k_{-1}$ в $(31)$, получаем

$$
k_{\mathrm{tot}}=\nu\left(F_{21}\right) \equiv\left\{e^{F_{21} \omega_{s_{0}}}+1\right\}^{-1} .
$$

Итак, константа скорости деканалирования (32) включает поперечную квазитемпературу КЧ в виде функции $\nu\left(F_{21}\right)$. Та же самая функция вошла в коэффициент деканалирования, полученный на основе обобщенных уравнений переноса [2], [17].

Область применимости реакции (30) не исчерпывается процессом деканалирования. Ее можно использовать при анализе широкого круга процессов, обусловленных случайными блужданиями частиц по уровням колебательной энергии между поглощающим и отражающим барьерами. В частности, реакция (30) описывает диссоциацию молекул в плазме. Большое отклонение системы от термодинамического равновесия в этом случае связано с незавершенностью колебательной релаксации, что в свою очередь приводит к значительному отрыву температуры колебательных степеней свободы от поступательной температуры [18]. Напомним, что в теории диссоциации [18] молекула рассматривается как осциллятор, который в результате столкновений с другими молекулами (осцилляторами) совершает переходы между 
колебательными уровнями. Если при этом молекула достигает уровня с энергией $E_{\mathrm{D}}$, равной энергии диссоциации, то последующее столкновение приводит к переходу ее из дискретного спектра в непрерывный, т.е. к распаду молекулы.

Учитывая различие термодинамических параметров в теории каналирования и диссоциации, из соотношения (32) можно выделить две ветви:

$$
k_{\mathrm{tot}}=\left\{\begin{array}{l}
\frac{1}{2} \frac{1}{F_{21} \omega_{s_{0}}}\left(\frac{1}{2}+\frac{1}{F_{21} \omega_{s_{0}}}\right)^{-1}, \\
e^{-E_{\mathrm{D}} / T_{V}} .
\end{array}\right.
$$

Первая ветвь соответствует деканалированию протонов $\left(E_{0} \sim 1 \mathrm{M \ni B}, 2 F_{21} \omega_{s_{0}}<1\right)$, вторая - диссоциации молекул в плазме $\left(F_{21} E_{\mathrm{D}} \equiv\left(E_{\mathrm{D}} / T_{V}\right)>1\right)$. Отметим, что во вторую ветвь константы скорости может дополнительно входить множитель, описывающий отставание $T_{V}$ от поступательной температуры. Кроме того, в условиях слабой дискретности спектра поперечной энергий КЧ диффузию частиц в поперечном энергетическом пространстве можно рассматривать как непрерывный безактивационный процесс. В то же время процесс диссоциации (вторая ветвь $k_{\text {tot }}$ ) является активированным.

\section{5. ЗАКЛЮЧЕНИЕ}

5.1. Квазитемпература как модуль квазиравновесного распределения. Выражения (16) и (26) - это квазиравновесные распределения в макроскопически малом объеме, внутри которого в данный момент времени находится быстрая частица. В этом объеме, точнее, внутри блока когерентных областей [19], устанавливается распределение, модулем которого является поперечная квазитемпература $1 / F_{21}(15)$. Остальной объем кристалла, исключая блок когерентных областей, остается в состоянии термодинамического равновесия с температурой $1 / \beta_{1}$, причем $1 / \beta_{1} \ll 1 / F_{21}$. В этой постановке задачи нет противоречия с общими принципами неравновесной термодинамики [3]; последняя допускает наличие в системе двух масштабов времени релаксации и, что особенно важно, малого времени установления квазиравновесия в макроскопически малом объеме.

Состояние системы можно описать не только набором средних величин $\left\langle P_{\min }\right\rangle$, но и набором дисперсий этих величин. Стационарное значение квазитемпературы $1 / F_{21}$ в принципе нетрудно связать со стационарной дисперсией поперечной энергии КЧ. Однако мы выбрали иной подход, основанный на уравнении баланса (12), что дало возможность раздельно вычислить вклад диффузии частиц в пространстве поперечной энергии ("разогрев" подсистемы $\{2\})$ и вклад диссипативного процесса (охлаждение подсистемы $\{2\}$ ). Если же пойти по пути прямого вычисления дисперсии, то учесть диссипацию энергии КЧ за счет увлечения электронов колеблющимся в канале рассеивающим центром [4] не представляется возможным.

Что касается зависимости угловых распределений от квазитемпературы (15), то высота максимума (20) распределения частиц, прошедших через кристалл, убывает с ростом $1 / F_{21}$, а минимальный выход $(29)$ в условиях обратного рассеяния растет. Учитывая пропорциональность квазитемпературы КЧ (15) температуре термостата, можно утверждать, что $I\left(0 ; 1 / F_{21}\right)$ уменьшается, а $\chi_{\min }\left(1 / F_{21}\right)$ увеличивается с 
ростом $1 / \beta_{1}$. Такой температурный ход наблюдался в экспериментальных исследованиях: в работе [20] - для высоты пика, в работах [21], [22] - для минимального выхода.

5.2. Приложение аналитической теории угловых распределений. Поперечная квазитемпература (15) доступна для экспериментального измерения, если использовать высоту пика углового распределения, полученного в рамках эксперимента "на прострел". Действительно, если наблюдаемое значение пика $I\left(0 ; 1 / F_{21}\right)$ подставить в выражение (20), то последнее трансформируется в трансцендентное уравнение для $1 / F_{21}$ (все остальные параметры в условиях конкретного эксперимента известны). Решение трансцендентного уравнения дает значение $1 / F_{21}$. Например, при прохождении ионов йода через кристалл серебра имеем $1 / F_{21}=6$ эВ (кристалл A), 1/ $F_{21}=13$ эВ (кристалл Б) (подробности см. в приложении). Высокие значения квазитемпературы являются следствием квазиупругого рассеяния КЧ на электронах, когда $\nu_{p_{\perp}} \gg \nu_{E_{\perp}}$, где $\nu_{p_{\perp}}-$ частота релаксации поперечного импульса КЧ.

\section{ПРИЛОЖЕНИЕ}

На рис. 1 представлено угловое распределение ионов йода, прошедших через кристалл серебра [9], [10]: толщина кристалла 0.835 мкм, энергия КЧ 12.6 МэВ, осевой канал [011], $\Phi_{h}=120$ эВ, $(-d E / d x)_{e}=3.7 \mathrm{MэB} /$ мкм, $f_{h}=0.31, \quad \psi_{\text {det }}=0.012$ град.

В таблице даны значения поперечной квазитемпературы КЧ, вычисленные на основе экспериментальных данных [9], [10]. Рассмотрено прохождение КЧ через два кристалла серебра (А и Б).

\begin{tabular}{|c|c|c|c|c|c|}
\hline & $\begin{array}{c}\text { Крити- } \\
\text { ческая } \\
\text { энергия } \\
\Phi_{h}, \text { эВ }\end{array}$ & $\begin{array}{c}\text { Крити- } \\
\text { ческий } \\
\text { угол } \\
\psi_{h}, \text { град. }\end{array}$ & $\begin{array}{c}\text { Угол раз- } \\
\text { упорядо- } \\
\text { чения } \\
\psi_{m}, \text { град. }\end{array}$ & $\begin{array}{c}\text { Высота } \\
\text { пика } \\
I\left(0 ; 1 / F_{21}\right)\end{array}$ & $\begin{array}{c}\text { Квазитем- } \\
\text { пература } \\
1 / F_{21}, \text { эВ }\end{array}$ \\
\hline $\begin{array}{c}\text { Кристалл А } \\
L=0.835 \text { мкм }\end{array}$ & 60 & 0.12 & 0.025 & $21.9 \cdot 10^{-4}$ & 6 \\
\hline $\begin{array}{c}\text { Кристалл Б } \\
L=0.775 \text { мкм }\end{array}$ & 120 & 0.14 & 0.022 & $2.3 \cdot 10^{-3}$ & 13 \\
\hline
\end{tabular}

\section{Список литературы}

[1] J. Lindhard. Mat-Fyz. Medd. Danske Vid. Selsk. 1965. V. 34. № 14. P. 1.

[2] Д. Н. Зубарев, Ю.А. Кашлев. ТМФ. 1976. Т. 29. № 3. С. 376.

[3] Д.Н. Зубарев. Неравновесная статистическая термодинамика. М.: Наука, 1971.

[4] Ю.А. Кашлев. ТМФ. 2004. Т. 138. № 1. С. 144.

[5] G. J. Kutcher, M.H. Mittleman. Phys. Rev. A. 1975. V. 11. № 1. P. 125.

[6] K.H. Mitchel. Physica. 1964. V. 30. № 4. P. 2194.

[7] F. Abel, G. Amsel, M. Bruneasux, C. Cohen, A.L'Heir. Phys. Rev. B. 1975. V. 12. № 11. P. 4617.

[8] Yu. A. Kashlev. Phys. Stat. Sol. B. 1995. V. 190. P. 379. 
[9] J.H. Barrett, B.R. Appleton, T.S. Noggle, C.D. Moak, J.A. Biggerstaf, S. Datz, R. Behrisch. Hyperchanneling. In: Proc. of the 5th Int. Conf. on Atomic Collisions in Solids (Gathingurg, Tennessee, September 24-28, 1973). Vol. 2. Eds. S. Datz, B. R. Appleton, C. D. Moar. N.Y.: Plenum, 1975. P. 645.

[10] B. R. Appleton, J.H. Barrett, T.S. Noggle, C.D. Moak. Rad. Eff. 1972. V. 13. P. 171.

[11] L. C. Feldman, B.R. Appleton. Phys. Rev. B. 1973. V. 8. № 3. P. 935.

[12] H.F. Krause, J.H. Barrett, S. Datz, P.F. Dittner, N.L. Jones, J. Gomes del Campo, C.R. Vane. Phys. Rev. A. 1994. V. 48. P. 283.

[13] B.R. Appleton, C. Erginsoy, W. M. Gibson. Phys. Rev. 1967. V. 161. P. 330.

[14] J. V. Andersen. Mat.-Fyz. Medd. Danske Vid. Selsk. 1967. V. 36. № 7. P. 1.

[15] J. A. Ellison. Phys. Rev. B. 1978. V. 18. № 11. P. 5948.

[16] Т. Келети. Основы ферментативной кинетики. М.: Мир, 1990.

[17] Ю.А. Кашлев. ТМФ. 1977. Т. 30. № 1. С. 82.

[18] А.И. Осипов. Теор. и экспер. химия. 1966. Т. 11. Вып. 5. С. 649.

[19] M. Rahman. Phys. Rev. B. 1995. V. 52. № 5. P. 3384.

[20] S. U. Campisano, G. Foti, E. Rimini. Nucl. Instr. Methods. 1976. V. 132. P. 169.

[21] K. Gartner, W. Wesch, G. Gotz. Nucl. Instr. Methods Phys. Res. B. 1990. V. 48. P. 192.

[22] J. H. Barrett, D. P. Jackson. Nucl. Instr. Methods. 1980. V. 170. P. 115.

Поступила в редакцию 26.IV.2005 г., после доработки 4.VII.2005 г. 